\title{
Impaired neutrophil activity and increased susceptibility to bacterial infection in mice lacking glucose-6-phosphatase- $\beta$
}

\author{
Yuk Yin Cheung, ${ }^{1,2}$ So Youn Kim, ${ }^{1}$ Wai Han Yiu, ${ }^{1}$ Chi-Jiunn Pan, ${ }^{1}$ Hyun-Sik Jun, ${ }^{1}$ Robert A. Ruef, ${ }^{1}$ \\ Eric J. Lee, ${ }^{3}$ Heiner Westphal, ${ }^{3}$ Brian C. Mansfield, ${ }^{1,4}$ and Janice Y. Chou ${ }^{1}$ \\ ${ }^{1}$ Section on Cellular Differentiation, Heritable Disorders Branch, National Institute of Child Health and Human Development (NICHD), NIH, \\ Bethesda, Maryland, USA. 2Department of Biochemistry, The Chinese University of Hong Kong, Hong Kong, People's Republic of China. \\ ${ }^{3}$ Laboratory of Mammalian Genes and Development, NICHD, NIH, Bethesda, Maryland, USA. ${ }^{4}$ Correlogic Systems Inc., Rockville, Maryland, USA.
}

\begin{abstract}
Neutropenia and neutrophil dysfunction are common in many diseases, although their etiology is often unclear. Previous views held that there was a single ER enzyme, glucose-6-phosphatase- $\alpha$ (G6Pase- $\alpha$ ), whose activity - limited to the liver, kidney, and intestine - was solely responsible for the final stages of gluconeogenesis and glycogenolysis, in which glucose-6-phosphate (G6P) is hydrolyzed to glucose for release to the blood. Recently, we characterized a second G6Pase activity, that of G6Pase- $\beta$ (also known as G6PC), which is also capable of hydrolyzing G6P to glucose but is ubiquitously expressed and not implicated in interprandial blood glucose homeostasis. We now report that the absence of G6Pase- $\beta$ led to neutropenia; defects in neutrophil respiratory burst, chemotaxis, and calcium flux; and increased susceptibility to bacterial infection. Consistent with this, G6Pase- $\beta$-deficient $\left(\mathrm{G} 6 \mathrm{pc}^{-/-}\right)$mice with experimental peritonitis exhibited increased expression of the glucose-regulated proteins upregulated during ER stress in their neutrophils and bone marrow, and the G6pc3 $3^{-/}$neutrophils exhibited an enhanced rate of apoptosis. Our results define a molecular pathway to neutropenia and neutrophil dysfunction of previously unknown etiology, providing a potential model for the treatment of these conditions.
\end{abstract}

\section{Introduction}

Quantitative or qualitative changes in neutrophil function are common in a number of diseases, such as glycogen storage disease type Ib (GSD-Ib; refs. 1-4), Shwachman-Diamond syndrome (5), and cyclic and autoimmune neutropenias (6-8), but the underlying cause is unclear. GSD-Ib is particularly interesting because although the molecular basis of the disease has been elucidated recently $(1,2)$ and clearly explains the predominant phenotype of disturbed glucose homeostasis, the connection to neutrophil dysfunction remains obscure. Moreover, a related disease, GSD-Ia (1), exhibits a metabolic phenotype nearly identical to that of GSD-Ib, but without the neutrophil dysfunction. Therefore, the 2 diseases provide an informative system with which to identify the cause of the neutrophil dysfunction. GSD-Ib is caused by mutations in the glucose-6-phosphate transporter (G6PT), which translocates glucose-6-phosphate (G6P) from the cytoplasm into the lumen of the ER, while GSD-Ia is caused by mutations in glucose- 6 -phosphatase- $\alpha$ (G6Pase- $\alpha$; also referred to as G6P catalytic subunit [G6PC]), which hydrolyzes endoluminal G6P to glucose $(1,2)$. Both G6Pase- $\alpha(9)$ and G6PT $(10)$ are ER transmembrane proteins, and their activities are functionally linked $(11,12)$. Therefore, a detrimental mutation in either protein prevents the other from functioning efficiently and leads to the same meta-

Nonstandard abbreviations used: $\mathrm{AMLP}$, f-Met-Leu-Phe; G6P, glucose-6-phosphate; G6Pase, glucose-6-phosphatase; G6PT, glucose-6-phosphate transporter; GRP, glucose-regulated protein; GSD-I, glycogen storage disease type I; H6PDH, hexose6-phosphate dehydrogenase; HSV-tk, herpes simplex virus thymidine kinase; KC, cytokine-induced neutrophil chemoattractant; MIP-2, macrophage inflammatory protein 2; neo, neomycin; UPR, unfolded protein response.

Conflict of interest: The authors have declared that no conflict of interest exists. Citation for this article: J. Clin. Invest. 117:784-793 (2007). doi:10.1172/JCI30443. bolic phenotype, manifested initially by changes in the glucose and lipid profiles of blood and in the longer term with kidney and liver disease $(1,2)$. While the metabolic abnormalities of GSD-Ia and GSD-Ib are almost identical (1), GSD-Ib patients exhibit neutrophil dysfunctions (1-4) not observed in GSD-Ia patients. The most noticeable difference between GSD-Ia and GSD-Ib that might explain this is the expression pattern of G6Pase- $\alpha$ and G6PT. G6Pase- $\alpha$ expression is restricted to the gluconeogenic organs of the liver, kidney, and intestine $(13,14)$, while G6PT is expressed ubiquitously (15), suggesting that G6PT might have different roles in gluconeogenic and nongluconeogenic tissues.

Recently a second G6Pase activity, that of G6Pase- $\beta$ (also known as G6PC3 or UGRP), was reported (16-18). The main difference between G6Pase- $\alpha$ and G6Pase- $\beta$ is that the latter shares a ubiquitous expression pattern (16) similar to that of G6PT (15). G6Pase- $\beta$ shares similar kinetic properties with G6Pase- $\alpha$ (17) and is an integral membrane protein in the $\mathrm{ER}$, containing 9 transmembrane domains (18) like G6Pase- $\alpha$ (9). The active site structures of G6Pase- $\alpha$ (19) and G6Pase- $\beta$ (18) are similar, and during G6P hydrolysis, both form a covalently bound phosphoryl-enzyme intermediate through a histidine residue, which lies on the luminal side of the ER membrane $(9,18)$. G6Pase- $\beta$ also couples functionally with the G6PT, in the same manner as G6Pase- $\alpha$ (17), to form an active G6Pase complex that hydrolyzes G6P to glucose. This suggests that the G6Pase- $\beta-$ G6PT complex might be functional in neutrophils and that the myeloid defects in GSD-Ib are caused by the loss of activity of that complex. We hypothesized that if this was found to be true in vivo, a knockout mutation of G6Pase- $\beta$ should exhibit the neutrophil dysfunctions of GSD-Ib but lack the metabolic abnormalities of both GSD-Ia and GSD-Ib. In order to test this hypothesis, we generated $\mathrm{G} 6 \mathrm{pc}^{-/-}$mouse 


\section{A}

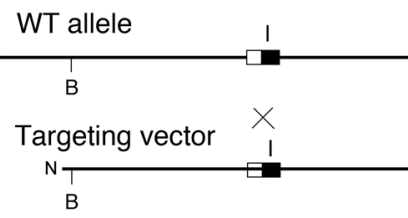

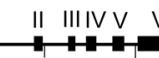

$\mathrm{Sd}$

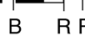

$B \quad R R$

II Neo VI

Sd RV B R

Sd RV B R R

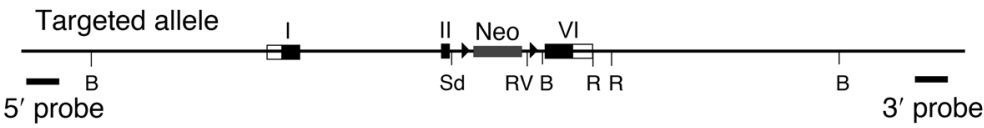

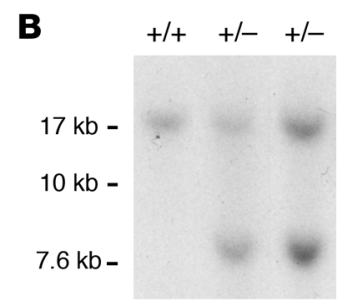

$5^{\prime}$ probe

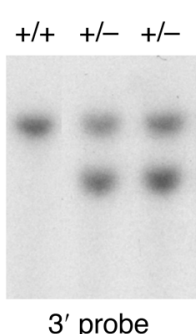

3 ' probe
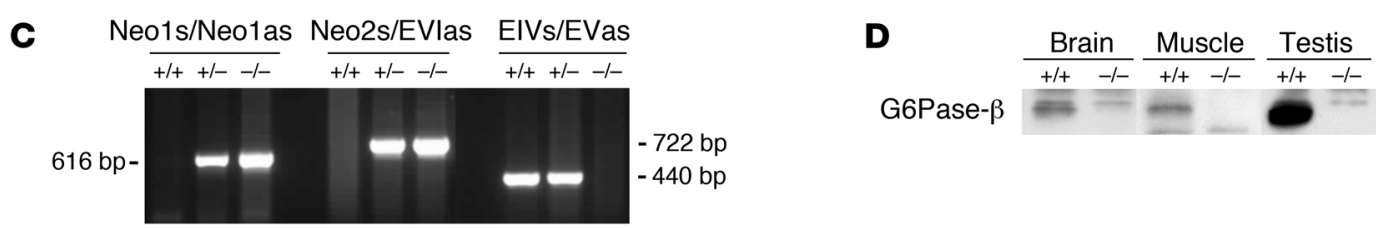

\section{Figure 1}

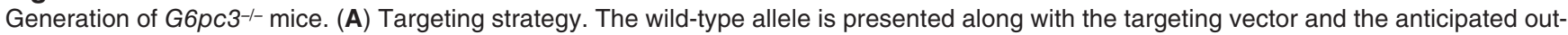
come of the recombination (targeted allele). Introns are denoted as lines, exons as boxes. Gene targeting resulted in the replacement of exons III-V with a loxP-flanked (arrowheads) neo cassette containing a diagnostic EcoRV site. The locations of the diagnostic $5^{\prime}$ and $3^{\prime}$ probes used in Southern blot analysis are shown. B, BamHI; N, Notl; R, EcoRI; RV, EcoRV; Sd, SanDI. (B) Southern blot analysis of genomic DNA from wild-type (+/+) and targeted ES (+/-) clones digested with EcoRV. (C) PCR analysis of genomic DNA of F1 intercross progeny. A wild-type locus-specific primer pair, EIVs/EVas, amplified a 440-bp fragment in the wild-type and heterozygous animals and 2 targeted locus-specific primer pairs, Neo1s/Neo2as and Neo2s/EVlas, amplified 616- and 722-bp fragments, respectively, in the heterozygous and homozygous $(-/-)$ animals. (D) Western blot analysis of brain, muscle, and testis microsomal protein preparations (50 $\mu \mathrm{g}$ of each tissue) using an antibody against human G6Pase- $\beta$ (48).

strains deficient in G6Pase- $\beta^{-/-}$by gene targeting. We showed that G6Pase- $\beta^{-/-}$mice manifested neutropenia and neutrophil dysfunctions mimicking those of GSD-Ib patients. We further showed that the expression of glucose-regulated proteins (GRPs), the ER chaperones known to be upregulated during ER stress (20-23), was significantly increased in the neutrophils and bone marrow of $G 6 p c 3^{-1-}$ mice during experimental peritonitis. Moreover, the G6pc3 $3^{-/-}$neutrophils exhibited a marked increase in apoptotic cell numbers compared with neutrophils from control littermate mice. Taken together, these results demonstrate that G6P translocation and metabolism in the ER are critical for normal neutrophil function and show that ER stress-mediated neutrophil apoptosis is one mechanism underlying myeloid dysfunction in $\mathrm{G} 6 \mathrm{pc}^{-/-}$mice.

\section{Results}

Generation of G6pc3-/- mice. The 129/SvJ mouse G6pc3 gene (encoding G6Pase- $\beta$ ), composed of 6 exons, was disrupted by replacement of exons III-V with a neomycin (neo) cassette and insertion into a targeting vector (24) containing a herpes simplex virus thymidine kinase (HSV-tk) for negative selection (Figure 1A). The construct was electroporated into J1 ES cells, and targeted clones were identified by Southern blot analysis of EcoRV-digested genomic DNA hybridized with a diagnostic 5' or 3' probe. The endogenous wild-type locus was characterized by a $17-\mathrm{kb}$ band with both probes, while the targeted ES cell locus yielded 17- and $7.6-\mathrm{kb}$ bands with the $5^{\prime}$ probe and 17 - and $10-\mathrm{kb}$ bands with the 3 ' probe (Figure 1B).

Two independent ES cell clones generated 2 lines of G6pc3-/mice carrying the G6Pase- $\beta$-null mutation, both with identical phenotypes. PCR of a 440-bp fragment in exons IV-V identified the wild-type gene, and PCR of a 616-bp fragment of the neo gene and a 722-bp fragment of the neo-exon VI fusion gene identified the disrupted gene (Figure 1C).
Western blot analysis of microsomal preparations of brain, muscle, and testis, tissues known to express high levels of G6Pase- $\beta$ (16), confirmed expression of the G6Pase- $\beta$ protein in wild-type mice and its absence in G6pc3-/- mice (Figure 1D). Phosphohydrolase assays showed significant activity in brain, muscle, and testis microsomes of wild-type mice, while background activities were detected in $G 6 p c 3^{-/-}$mice (Table 1).

The G6pc3 $3^{-1-}$ animals were born at the expected Mendelian frequency and were morphologically indistinguishable from wildtype mice. The liver/kidney/intestine G6Pase- $\alpha$ is the dominant hydrolase in glucose homeostasis (1); consistent with this, the G6pc3 $3^{-1-}$ mice exhibited none of the metabolic deficiencies typical of GSD-Ia and GSD-Ib. The body weights of G6pc3 $3^{-/}$animals were comparable to those of age-matched wild-type mice. The G6pc3-/livers and kidneys were of normal size, and there was no abnormal glycogen accumulation in either organ (data not shown). Also, the G6pc3 $3^{-1-}$ mice exhibited blood glucose, triglyceride, cholesterol, uric acid, and lactic acid profiles comparable to those of wild-type littermates (Figure 2A). Therefore, a deficiency in G6Pase- $\beta$ does not disrupt glucose homeostasis.

G6pc3 $3^{-/-}$mice manifest neutropenia and neutrophil dysfunctions. G6pc3 $3^{-/-}$ is considered a ubiquitously expressed gene (16), but expression of

\section{Table 1}

Microsomal G6P phosphohydrolase activity

$\begin{array}{lrl} & \text { Wild-type } & \text { G6pc } \text { - }^{--} \\ \text {Brain } & 9.5 \pm 0.9 & 1.4 \pm 0.3^{\mathrm{A}} \\ \text { Muscle } & 5.1 \pm 0.5 & 1.5 \pm 0.4^{\mathrm{A}} \\ \text { Testis } & 15.0 \pm 0.5 & 0.9 \pm 0.8^{\mathrm{A}}\end{array}$

Values (mean \pm SEM) are reported as $\mathrm{nmol} / \mathrm{mg} / \mathrm{min}$. ${ }^{A} P<0.01$ versus wild-type. 

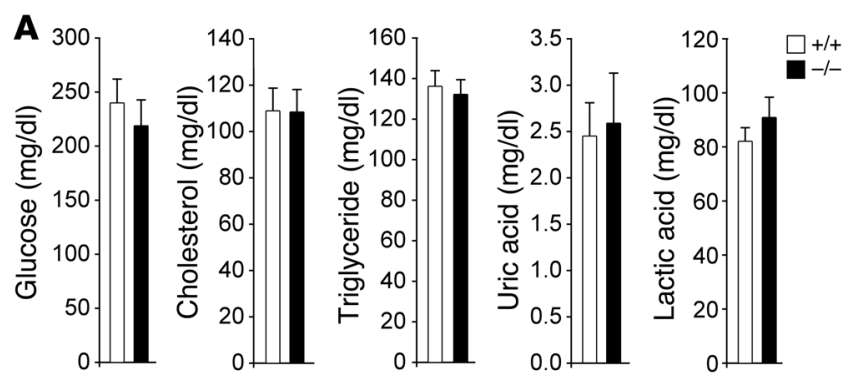

B

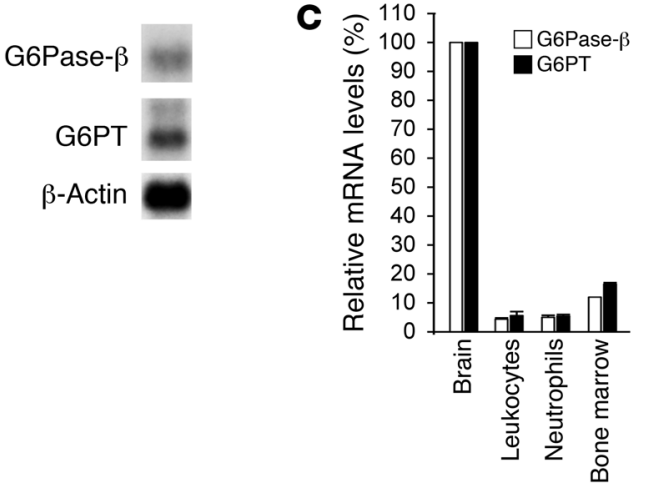

this gene specifically in neutrophils and bone marrow, the latter of which is the primary site of maturation and development of hematopoietic cells (25), has not to our knowledge been previously demonstrated. Northern blot analysis showed that human blood neutrophils, which express nondetectable levels of the gene encoding for G6Pase- $\alpha$ (15), expressed both G6PT and G6Pase- $\beta$ transcripts (Figure $2 \mathrm{~B}$ ). Real-time PCR analysis was used to measure the expression levels of mouse genes encoding for G6Pase- $\beta$ and G6PT in RNA isolated from brains, blood leukocytes, peritoneal neutrophils, and bone marrow of wild-type mice, and the expression levels were normalized by the level of G6Pase- $\beta$ transcript in the brain, which was arbitrarily defined as $100 \%$. Mouse leukocytes, neutrophils, and bone marrow expressed both G6Pase- $\beta$ and G6PT mRNA at similar relative levels (Figure 2C).

We next sought to determine whether a knockout mutation of G6Pase- $\beta$ would lead to the neutrophil dysfunctions of GSD-Ib, characterized by neutropenia accompanied by impaired neutrophil respiratory burst activity, chemotaxis, and $\mathrm{Ca}^{2+}$ flux response $(1-4,26)$. The G6pc3 ${ }^{-/-}$mice were neutropenic. Differential peripheral blood leukocyte counts for neutrophils in 3-week-old G6pc3-/mice averaged only $36 \%$ of those for age-matched wild-type littermates (Figure 3A). Like GSD-Ib mice (26), neutropenia improved with age, but neutrophil counts for 6- to 7-week-old G6pc3 $3^{--}$mice still only averaged $67 \%$ of those in controls (Figure $3 \mathrm{~A}$ ).

Intraperitoneal injection of thioglycollate into 6- to 7-week-old wild-type mice led to the recruitment of large numbers of neutrophils into the peritoneal space (Figure 3B). In contrast, peritoneal neutrophil accumulation in the $G 6 \mathrm{pc}^{-/-}$peritoneum was decreased to $52 \%$ of that in controls (Figure 3B). Therefore, the G6pc3 $3^{-/-}$mice exhibit a defect in neutrophil trafficking in vivo during an inflammatory response similar to the defect in neutrophil accumulation previously observed in GSD-Ib mice, in which neutrophil accumulation in the G6PT-deficient peritoneum decreased to 33\% that of controls (26).

The percentages of neutrophils in the peritoneal cell populations were similar between $G 6 p c 3^{-/-}(81.8 \% \pm 4.7 \%)$ and wild-type

\section{Figure 2}

Analysis of the serum profiles and expression of G6Pase- $\beta$ and G6PT mRNA. (A) Serum concentrations of glucose, cholesterol, triglyceride, uric acid, and lactic acid in wild-type and $\mathrm{G} 6 \mathrm{pc} 3^{-/-}$mice. Fourteen mice of each genotype were used. Values represent mean \pm SEM. (B) Northern blot analysis of human G6Pase- $\beta$ and G6PT mRNA expression in human neutrophils. $\beta$-Actin was used as a control. Each lane contained $3 \mu \mathrm{g}$ poly (A)+ RNA. (C) Quantification of murine G6Pase- $\beta$ and G6PT mRNA by real-time RT-PCR in 6-week-old wild-type mice. Leukocytes were isolated from the whole blood, and neutrophils were isolated from thioglycollate-induced peritoneum. Expression levels are shown relative to that of G6Pase- $\beta$ or G6PT transcript in the brain, which was arbitrarily assigned as $100 \%$.

littermates $(84.3 \% \pm 5.8 \% ; P=0.75)$, and both populations were composed of morphologically mature neutrophils, including band and segmented nucleophilic cells (Figure 3C). The G6pc3-/- and wild-type neutrophils also expressed similar levels of the granule protein gelatinase and the myeloid differentiation antigen Gr-1 (Figure 3D). Because gelatinase is a marker of terminal neutrophil differentiation $(27,28)$ and $\mathrm{Gr}-1$ is a marker of neutrophil maturation $(29,30)$, these results indicate that neutrophils in both peritoneal cell populations are of similar maturity.

Respiratory burst activity was assessed in thioglycollate-recruited peritoneal neutrophils from $G 6 p c 3^{-/-}$and wild-type littermates. In wild-type mice, superoxide production in neutrophils was markedly increased by exposure to phorbol myristate acetate (PMA) (Figure 3E). However, for G6pc3-/- mice, PMA-stimulated superoxide production was reduced in neutrophils (Figure $3 \mathrm{E}$ ), implying that G6Pase- $\beta$ expression in neutrophils is important for respiratory burst activity.

Thioglycollate-elicited peritoneal neutrophils from wild-type mice exhibited a greater dose-dependent chemotactic response to the bacterial peptide f-Met-Leu-Phe (fMLP) as well as the chemokines cytokine-induced neutrophil chemoattractant (KC) and macrophage inflammatory protein 2 (MIP-2) than did neutrophils from $G 6 p c 3^{-1-}$ mice (Figure $4 \mathrm{~A}$ ), which suggests that chemotaxis is impaired in the $\mathrm{G} 6 \mathrm{pc}^{-/-}$mice. In wild-type mice, mobilization of $\mathrm{Ca}^{2+}$ increased in response to $\mathrm{MMLP}, \mathrm{KC}$, and MIP-2, whereas $\mathrm{Ca}^{2+}$ mobilization was impaired in $\mathrm{G}_{6} \mathrm{pc}^{-/-}$neutrophils (Figure $4 \mathrm{~B})$. Therefore, a deficiency of G6Pase- $\beta$ in neutrophils also led to problems regulating intracellular $\mathrm{Ca}^{2+}$ concentrations. We conclude that $\mathrm{G} 6 \mathrm{pc}^{-/-}$mice exhibit impaired neutrophil respiratory burst, chemotaxis, and $\mathrm{Ca}^{2+}$ flux activities similar to those of neutrophils from GSD-Ib patients (1-4) and mice (26).

G6pc3 $3^{-1-}$ mice exhibit increased susceptibility to bacterial infection. The neutropenia and neutrophil dysfunctions in GSD-Ib patients usually result in recurrent bacterial infections (1-4). In order to determine whether G6pc3-/- mice exhibit similar bacterial susceptibility, we used a murine model of acute lethal bacterial infection. G6pc3-1- and wild-type littermates were challenged with a lethal intraperitoneal dose of Escherichia coli $\left(4 \times 10^{7} \mathrm{CFU} / 30 \mathrm{~g}\right)$, and survival rates were compared (Figure 5A). Infected G6pc3 $3^{-1-}$ mice showed increased susceptibility, with $92 \%$ (22 of 24) of knockout mice dying after 20 hours of infection compared with $29 \%$ (7 of $24)$ of wild-type littermates. The surviving $2 \mathrm{G} 6 \mathrm{pc} 3^{-1-}$ mice recovered completely from the infection, and 9 of the 24 wild-type littermates recovered completely (Figure 5A).

The number of viable bacteria in the blood of the challenged G6pc3-1- and wild-type mice 5 hours after infection correlated to 
A

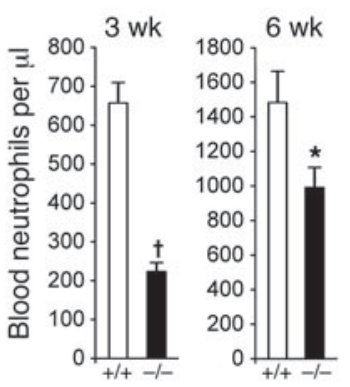

D

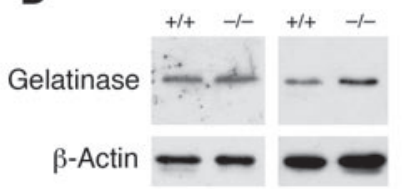

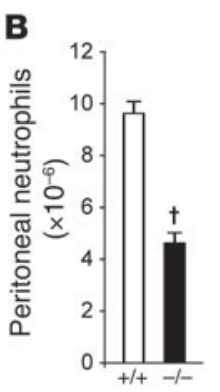
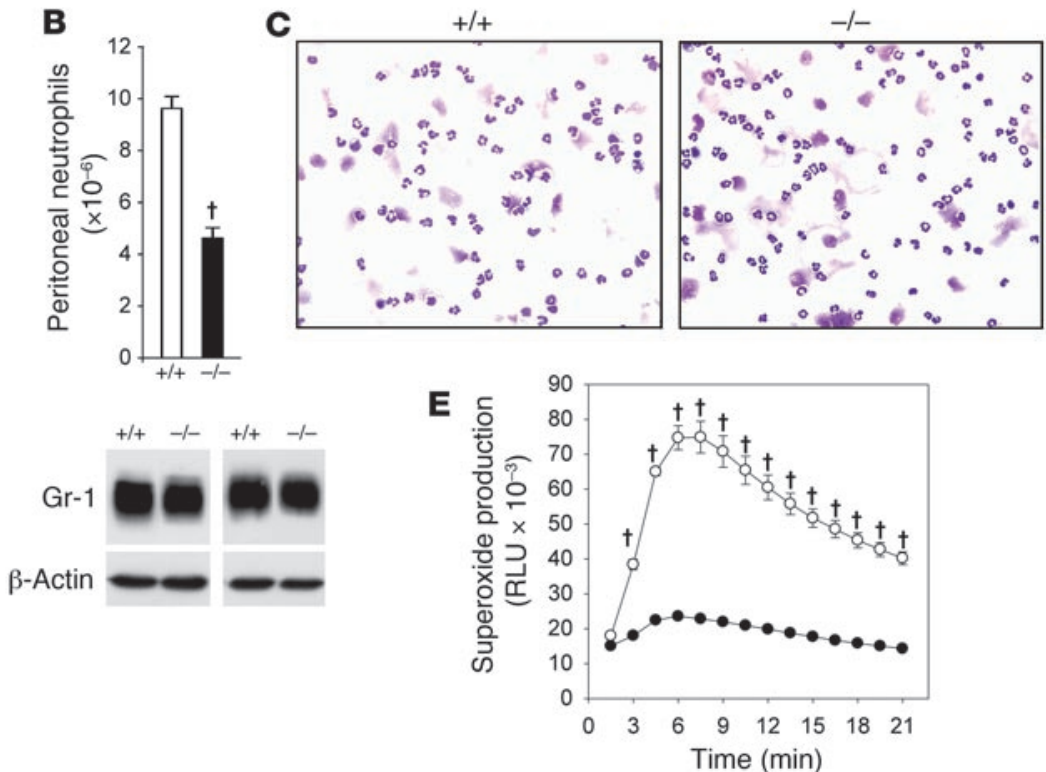

Figure 3

Analysis of myeloid functions. (A) Neutrophil counts in 3-week-old $(n=8)$ and 6-week-old $(n=12)$ wild-type and G6pc $3^{-/-}$mice. (B) Total peritoneal neutrophil counts in 6- to 7-week-old wild-type $(n=15)$ and G6pc3-/- $(n=16)$ mice challenged with thioglycollate. (C) Hema 3-stained cytospins of peritoneal neutrophils obtained from 7-week-old wild-type and G6pc3-/- mice. (D) Western blot analysis of protein extracts of peritoneal

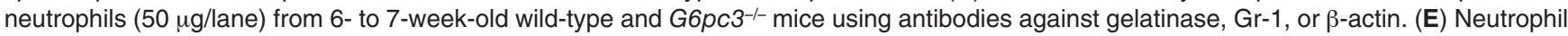
respiratory burst activity. Results represent triplicate determinations using peritoneal neutrophils from 6- to 7-week-old wild-type (open circles) or G6pc3-/- (filled circles) mice. RLU, relative light unit. Values are mean \pm SEM. ${ }^{*} P<0.03$; ${ }^{\top} P<0.0005$ versus wild-type.

survival time (Figure 5B). Mice dying early in infection showed considerably higher concentrations of viable bacteria in their blood than did those dying late in infection. Mice that survived the bacterial challenge showed low or undetectable levels of viable bacteria in their blood 5 hours after infection.

G6pc3 $3^{-1}$ mice do not exhibit impaired hematopoiesis in bone marrow. Impaired hematopoiesis in the bone marrow could be the cause of the neutropenia and neutrophil dysfunction we observed in G6pc3 $3^{-1-}$ mice. We therefore examined hematopoiesis in $\mathrm{G} 6 \mathrm{pc} 3^{-1-}$ and wild-type littermates. The total number of cells in the bone marrow aspirates combined from the femur and tibia were similar between $\mathrm{G} 6 \mathrm{pc}^{-/-}$and wild-type mice (data not shown), and both aspirates expressed similar levels of gelatinase $(27,28)$ and $\mathrm{Gr}-1$ $(29,30)$ (Figure 6A). Interestingly, G6pc3 $3^{-/}$aspirates contained a slightly higher proportion of CFUs than did wild-type aspirates (Figure 6B). In bone marrow aspirates from 3-week-old mice, in vitro clonal stimulation with G-CSF, GM-CSF, or M-CSF caused granulocyte, granulocyte-macrophage, and macrophage CFUs to increase 1.6-, 1.6-, and 1.7-fold, respectively, in G6pc3-/- mice compared with wild-type littermates (Figure 6B). By 7 weeks of age, this had not changed significantly: the counts of granulocyte, granulocyte-macrophage, and macrophage CFUs increased 1.8-, $1.3-$, and 1.5-fold, respectively, in G6pc3 $3^{-1-}$ mice compared with wild-type littermates (Figure 6B). Moreover, $40 \%-45 \%$ of granulocyte CFUs from $\mathrm{G}_{\mathrm{C}} \mathrm{C3}^{-/-}$and control littermates were morphologically mature neutrophils (Figure 6C). Taken together, these data strongly suggest that the myeloid dysfunction manifested by G6pc3 $3^{-1-}$ mice is not caused by impaired granulocyte differentiation or a decrease in the number of myeloid progenitor cells.

Increased GRP expression in the neutrophils and bone marrow of G6pc3 $3^{-1-}$ mice. The myeloid dysfunctions seen in the G6pc3 $3^{-1-}$ mice support the hypothesis that endoluminal glucose production from G6P hydrolysis, catalyzed by G6Pase- $\beta$, is required for normal neutrophil function. The lumen of the ER serves as a critical site in protein maturation, and its biochemical environment is uniquely designed to facilitate optimal posttranslational modification, folding, and assembly of proteins destined for the cell membrane or for secretion (31). Glucose deprivation results in perturbations of the internal environment of the ER, thereby compromising its functions $(20,21)$. This in turn triggers the unfolded protein response (UPR), which is characterized by the increased synthesis of GRPs, the ER chaperones that assist in protein folding and assembly (20-23). If endoluminal glucose production is critical for ER homeostasis, then in an inflammatory response GRP expression should be increased in neutrophils and/or bone marrow in $G 6 p c 3^{-1-}$ mice relative to wild-type littermates. Indeed, during thioglycollate-elicited peritonitis, the expression of GRP78 (also referred to as Bip; refs. 20-23) and GRP170 (21-23) mRNA in both neutrophils (Figure 7A) and bone marrow (Figure 7B) of G6pc3 $3^{-1-}$ mice was markedly elevated over that of their wild-type littermates. Western blot analysis confirmed the increase in GRP proteins in neutrophils (Figure 7C) and bone marrow (Figure 7D) of $\mathrm{G} 6 \mathrm{pc3^{-1- }}$ mice compared with wild-type controls.

G6pc3 $3^{-1-}$ mice exhibit an enhanced rate of neutrophil apoptosis. The increase in the expression of GRPs suggests that the G6pc3-/- neutrophils undergo ER stress. Because prolonged ER stress triggers cell death, usually in the form of apoptosis $(32,33)$, we examined 2 apoptotic markers in $\mathrm{G} 6 \mathrm{pc}^{-/-}$neutrophils, the externalization of phosphatidylserine (34) and the activation of caspase-3 $(35,36)$. The maintenance of membrane asymmetry is inherent to healthy animal cells, and a breakdown of this asymmetry indicates that cells have entered the early to middle stages of apoptosis $(34,37)$, 

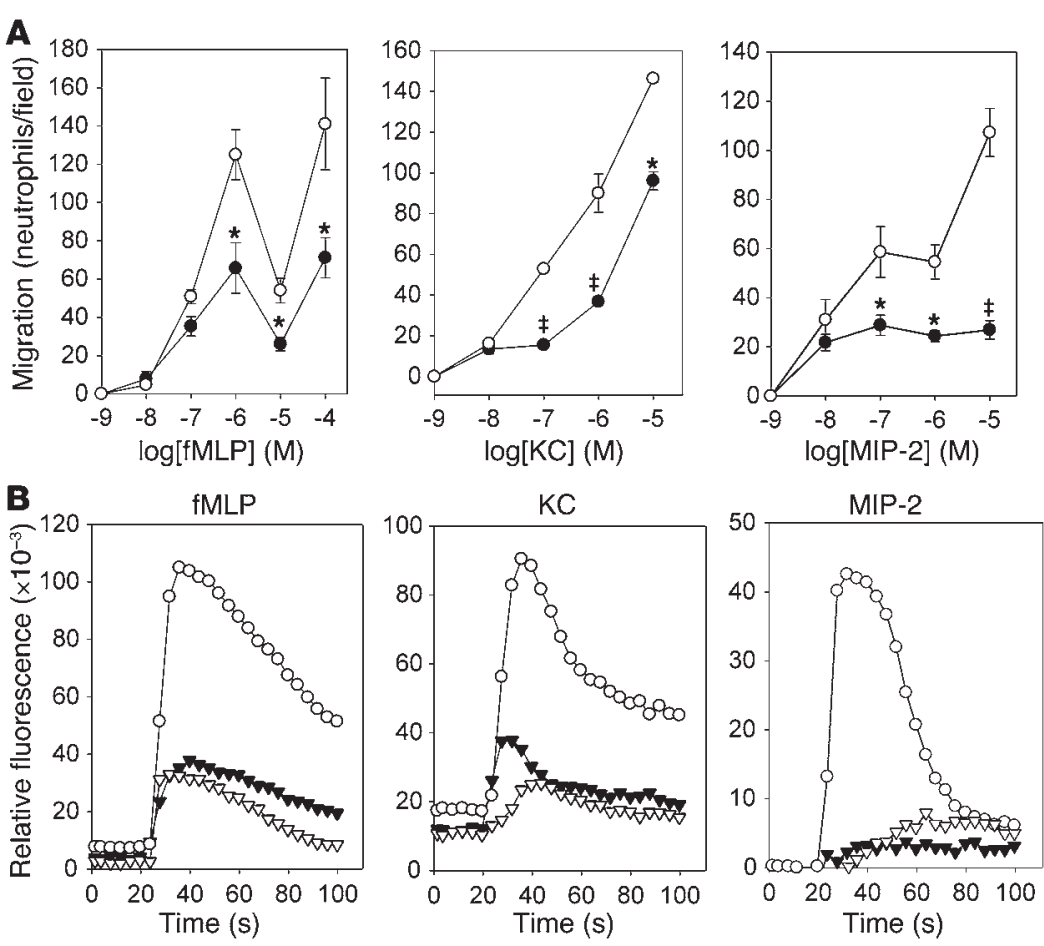

\section{Figure 4}

Impaired chemotaxis and calcium flux of $\mathrm{G} 6 p c 3^{-/-}$ neutrophils in response to fMLP, KC, and MIP-2. Neutrophils were isolated from thioglycollate-induced peritoneum of 6- to 7-week-old mice. (A) Concentration-dependent chemotaxis. Open circles, wild-type; filled circles, G6pc3--. Values represent mean \pm SEM of quadruplet determinations. ${ }^{*} P<0.03$, $¥ P<0.005$ versus wild-type. (B) $\mathrm{Ca}^{2+}$ flux in response to $10^{-6} \mathrm{M}$ fMLP, KC, or MIP-2. Representative experiments are shown. Open circles, wild-type; open and filled triangles, both $\mathrm{G} 6 \mathrm{pc} 3^{-/}$.

to glucose in the terminal step of gluconeogenesis and glycogenolysis. This process is catalyzed by a G6Pase complex, which is composed of a ubiquitously expressed G6PT and a G6Pase catalytic unit, more recently described as G6Pase- $\alpha(1,2)$. In order to be active, G6Pase and G6PT have to couple functionally. Until recently, G6Pase activity was thought to be confined to the liver, kidney, and intestine $(13,14)$, the only tissues known to contain G6Pase- $\alpha$. Most other tissues and organs in the body were considered unable to generate glucose because of the absence of a G6Pase to couple to the ubiquitous G6PT. Indeed, human patients $(1-4)$ and mice $(11,26)$ deficient in either

which can be scored by annexin $\mathrm{V}$ binding to externalized phosphatidylserine (38), one of the principal inner membrane phospholipid components of the phospholipids bilayer (34). The caspases, which are activated through proteolysis at specific asparagine residues, play a central role in the regulation and execution of apoptotic cell death (reviewed in refs. 35,36 ). Caspase- 3 is one of the effector caspases that initiate the death pathways induced by diverse stimuli. In G6pc3-/- mice $(n=4), 8 \%-12 \%$ of peritoneal neutrophils stained positive for annexin $\mathrm{V}$, while few, if any, neutrophils from wildtype littermates bound to annexin $\mathrm{V}$ (Figure 8A), which suggests an enhanced rate of apoptosis in the $\mathrm{G} 6 \mathrm{pc3^{-/- }}$ neutrophils. Consistent with this, the expression of caspase-3 mRNA in G6pc3 $3^{-/-}$neutrophils was elevated compared with that of wild-type neutrophils (Figure 8B). Moreover, Western blot assays (Figure 8C) and activity assays (Figure 8D) demonstrated that levels of active caspase-3 were markedly increased in $G 6 p c 3^{-/-}$neutrophils.

\section{Discussion}

Glucose homeostasis between meals is maintained by endogenous glucose that is produced by the dephosphorylation of G6P
G6Pase- $\alpha$ (i.e., GSD-Ia) or G6PT (i.e., GSD-Ib) are unable to maintain glucose homeostasis. However, G6PT deficiency has the additional phenotype of neutrophil dysfunctions that cannot be readily explained by the G6PT defect. The recent recognition of a second G6Pase activity, that of G6Pase- $\beta$ (16-18), and its universal expression profile (16) raised the possibility that endogenous glucose is produced to some extent in many tissues through the coupling of G6Pase- $\beta$ with G6PT. Because it is well established that G6Pase- $\alpha$ is the key enzyme in interprandial blood glucose homeostasis (1), G6Pase- $\beta-$ G6PT complex-mediated glucose production is not expected to be important for normal blood glucose homeostasis. We hypothesized that G6Pase- $\beta$ activity could become critical in the ER during periods of stress and that the myeloid dysfunctions observed in GSD-Ib may be caused, at least in part, by the loss of G6Pase- $\beta-$ G6PT complex activity. In this study, we showed that mice deficient in G6Pase- $\beta$ exhibited no disturbance in blood glucose homeostasis but did suffer neutropenia; defects in neutrophil respiratory burst, chemotaxis, and calcium flux; and increased susceptibility to bacterial infection. Our study demonstrates, for the first time to our knowledge, that G6Pase- $\beta$ activity is critical for

\section{Figure 5}

Susceptibility of $\mathrm{G}_{\mathrm{f}} \mathrm{pc} \mathrm{3}^{-/-}$mice to bacteria infection. (A) Survival curves and (B) viable blood-born bacteria at 5 hours after infection for wild-type (open circles) and G6pc $^{-/-}$(filled circles) mice $(n=24$ per group) challenged with $E$. coli at $4 \times 10^{7}$ CFU/30 g body weight. Results are from 2 independent experiments. $P<0.0001$ for survival curves, log-rank test.
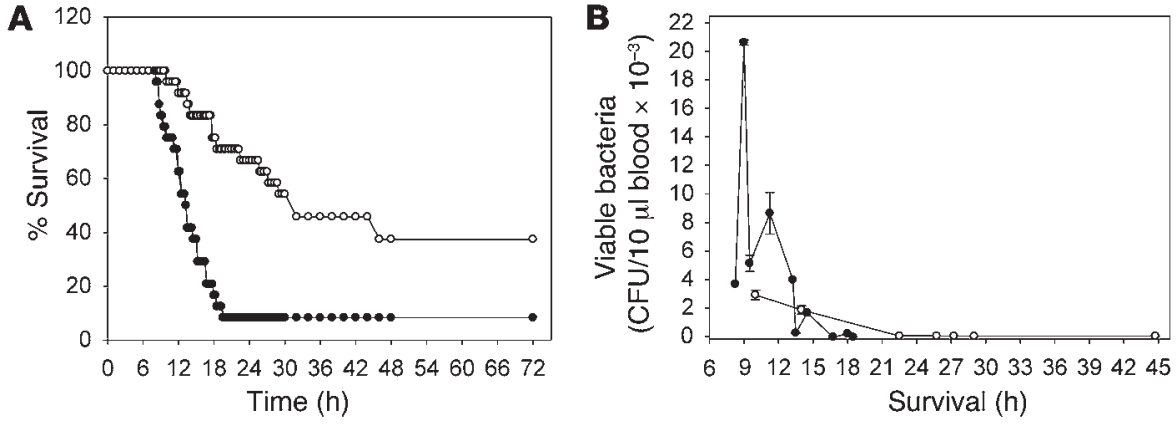
A

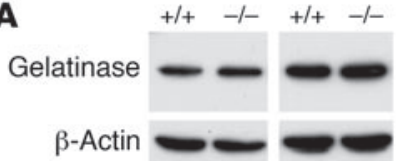

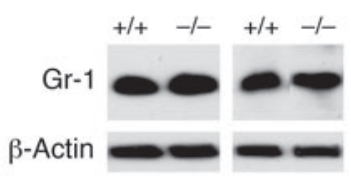

B
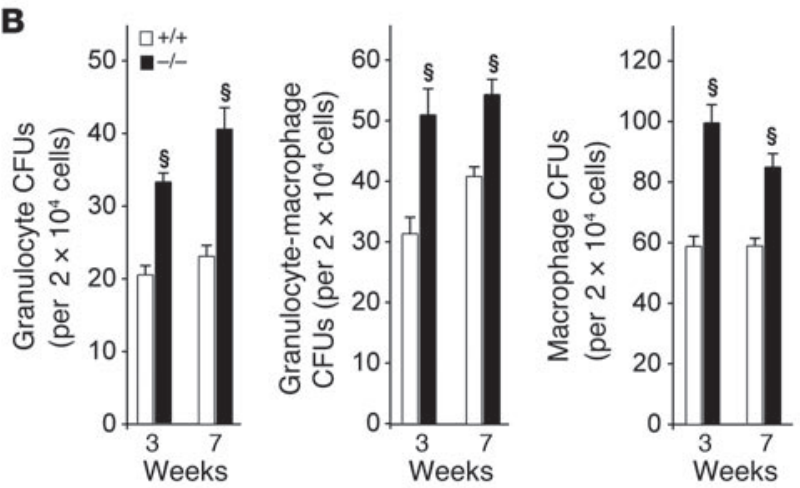

C
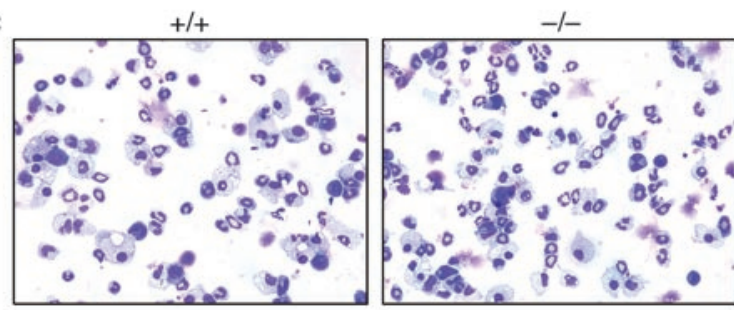

normal neutrophil and bone marrow function, and we propose that the lack of a functional G6Pase- $\beta-$ G6PT complex causes the neutropenia and myeloid dysfunctions that characterize GSD-Ib.

G6PT (10), G6Pase- $\beta$ (18), and G6Pase- $\alpha$ (9) are colocalized in the membrane of the ER, embedded by multiple transmembrane domains. The most obvious and well-documented role of G6PT is in the gluconeogenic tissues of the liver and kidney, where it transports G6P from the cytoplasm into the lumen of the ER for hydrolysis to glucose by G6Pase- $\alpha(1,2)$. Recent bone marrow transplantation studies show that wild-type mice transplanted with bone marrow of G6PT-deficient animals manifest neutropenia along with myeloid dysfunctions in $\mathrm{Ca}^{2+}$ mobilization, respiratory burst, and chemotaxis, demonstrating that G6PT expression in bone mar-

\section{Figure 6}

Analysis of hematopoiesis. (A) Western blot analysis of bone marrow

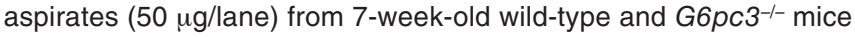
using antibodies against gelatinase, Gr-1, or $\beta$-actin. (B) Myeloid progenitor cells in the femur plus tibia of wild-type and $\mathrm{G} 6 \mathrm{pc} 3^{-/-}$mice at 3 and 7 weeks of age. CFUs were determined following stimulation of bone marrow cells with G-CSF, GM-CSF, or M-CSF. Results are mean \pm SEM from 4 separate experiments, in which each mouse was assessed individually. $\$ P<0.001$ versus wild-type. (C) Hema 3stained cytospins of granulocyte CFUs obtained from 3-week-old wildtype and $\mathrm{G} 6 \mathrm{pc} 3^{-/-}$mouse bone marrow cells following 9 days' stimulation with G-CSF.

row and neutrophils is required for normal myeloid function (39). We now show that $G 6 p c 3^{-/-}$mice, unable to hydrolyze endoluminal G6P to glucose, manifest myeloid dysfunctions mimicking G6PT deficiency. This supports the hypothesis that the absence of a functional G6Pase- $\beta-$ G6PT complex can be caused by the loss of activity of either component. The absence of either G6Pase- $\beta$ or G6PT then results in the loss of endogenous glucose production in neutrophils and bone marrow and leads to their dysfunction.

There is accumulating evidence that the phenotype of null mutations can be modified by differing genetic backgrounds $(40,41)$, particularly when examining the severity of the immune response to bacterial challenge $(42,43)$. In the present study, the F2 mice had backgrounds contributed from both the C57BL/6 and 129/SvJ strains, and we do not directly rule out the effects this might have had on our bacterial challenge experiments. However, both the G6pc3 $3^{-1-}$ mice studied here and the G6PT-deficient mice reported previously (26) have a similar C57BL/6 and 129/SvJ hybrid background. The G6pc3 $3^{--}$mice had the same myeloid phenotype seen in both G6PT-deficient mice and G6PT-deficient human GSD-Ib patients, which suggests that the neutropenia, neutrophil dysfunctions, and increased susceptibility to bacterial infection manifested by the G6pc3 $3^{--}$mice were the direct result of the disruption of the G6Pase- $\beta$ complex rather than a genetic background effect. However, to address this aspect directly, we are currently generating the coisogenic and congenic G6pc3 $3^{--}$lines and will use the F1 hybrid offspring to further delineate myeloid dysfunctions in the G6pc3 $3^{-/}$mice.

There are a number of potential mechanisms that could account for myeloid dysfunction, such as impaired granulocytic differentia-
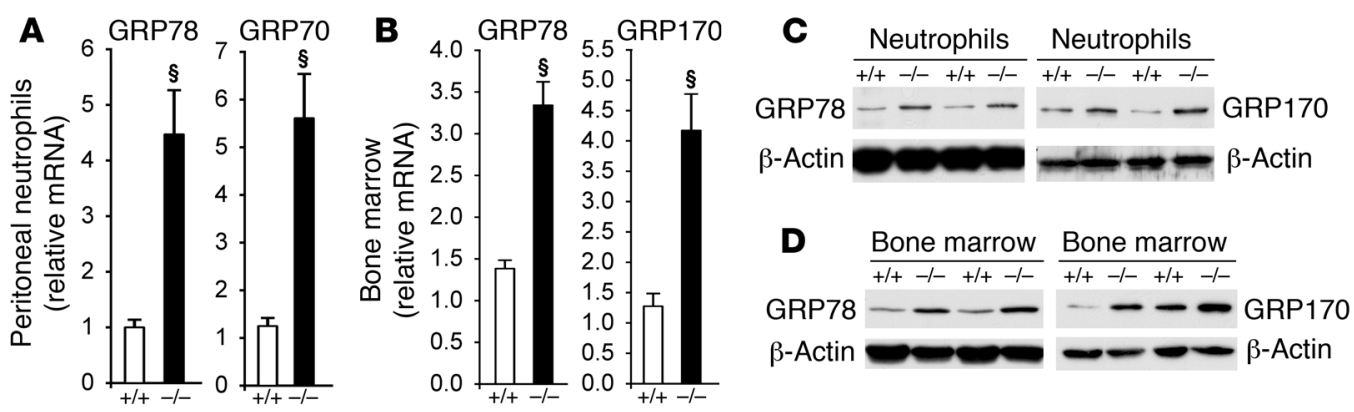

Figure 7

Real-time PCR and Western blot analyses of GRP gene expression. Peritoneal neutrophils and bone marrow aspirates were isolated from 6- to 7-week-old wild-type and G6pc3-/- mice during thioglycollate-elicited peritonitis. (A and B) Real-time PCR analysis. The expression levels of the GRP78 and GRP170 transcripts were normalized to $\beta$-actin RNA and then scaled relative to the transcript in a wild-type littermate, arbitrarily assigned as 1. (A) Peritoneal neutrophils ( $n=10$ and 13 for wild-type and G6pc3 $3^{-/-}$, respectively). (B) Bone marrow ( $n=14$ and 18 for wildtype and G6pc $3^{-/-}$, respectively). Values represent mean \pm SEM. $\$ P<0.001$. (C and D) Immunoblot analysis of protein extracts of neutrophils (50 $\mu \mathrm{g} / \mathrm{lane} ; \mathbf{C}$ ) and bone marrow (D) using antibodies against GRP78, GRP170, or $\beta$-actin. 
A
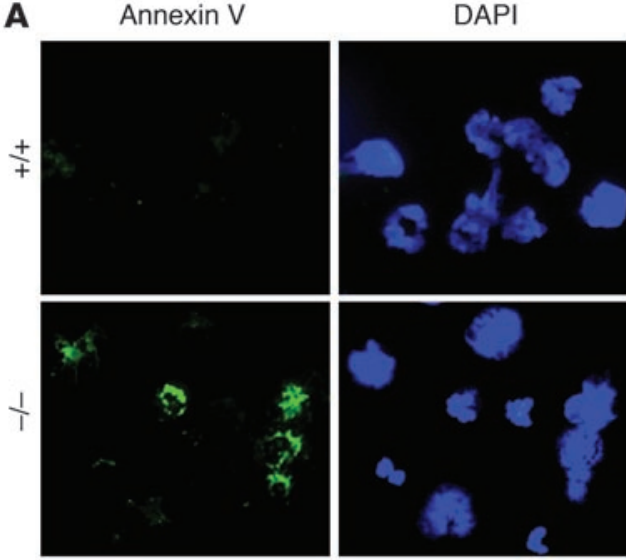

B

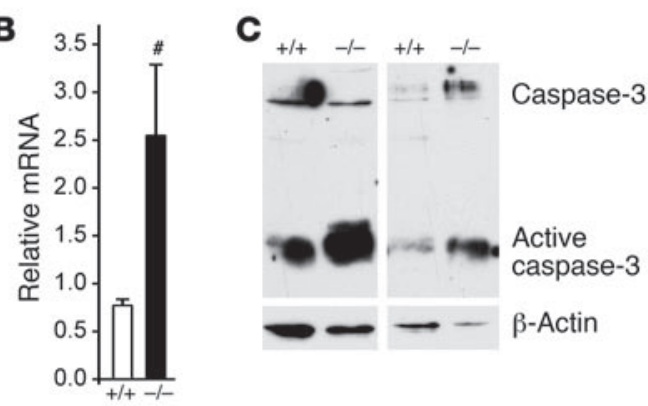

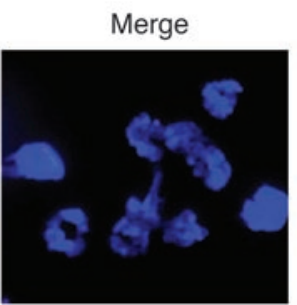
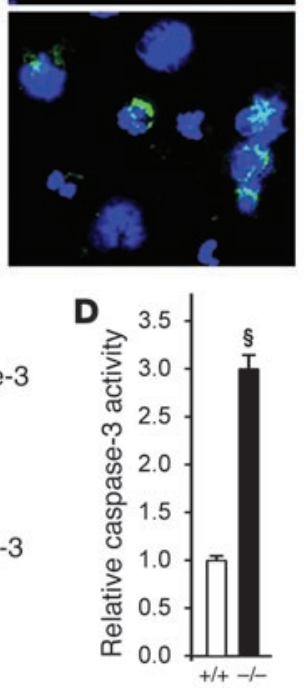

Figure 8

Analysis of neutrophil apoptosis. Peritoneal neutrophils were isolated from 6- to 7-week-old wild-type and G6pc3-/- mice during thioglycollate-elicited peritonitis. (A) Immunofluorescence of annexin $V$ surface staining of peritoneal neutrophils (green fluorescence) and DAPI staining of nuclei (blue fluorescence). Note the colocalization of annexin V staining with DAPI staining in the merged image. Original magnification, $\times 200$. (B) Expression of the caspase- 3 transcript in wild-type and G6pc $3^{-/-}$mice ( $n=12$ per group) quantified by real-time PCR. The expression levels of the caspase-3 transcript were normalized to $\beta$-actin RNA and then scaled relative to the transcript in a wild-type littermate, arbitrarily assigned as 1 . Values represent mean \pm SEM. ${ }^{\#} P=0.016$. (C) Immunoblot analysis of protein extracts of neutrophils $(50 \mu \mathrm{g} / \mathrm{lane})$ using antibodies against caspase- 3 or $\beta$-actin. (D) DEVD-cleaving activity of active caspase- 3 in protein extracts of peritoneal neutrophils from wild-type and $\mathrm{G} 6 \mathrm{pc} 3^{-/-}$mice ( $n=3$ per group). Activities were normalized to that of wild-type caspase-3. Values represent mean \pm SEM. ${ }^{\S} P<0.001$.

in annexin $\mathrm{V}$ binding and caspase-3 activation (Figure 8). This suggests that at least one mechanism of the myeloid dysfunction in $\mathrm{G}_{6 \mathrm{pc}} 3^{-/-}$mice involves enhanced neutrophil apoptosis caused by the disruption of glucose production in the ER. It is reasonable to expect that a similar mechanism may be responsible for the myeloid dysfunction in GSD-Ib patients and in GSD-Ib mice.

G6Pase- $\beta$, like G6Pase- $\alpha$, is linked functionally with G6PT tion and/or decreased myeloid progenitor cell numbers, for which we observed no supporting evidence. Bone marrow aspirates from G6pc3 $3^{-/}$and wild-type littermates expressed similar levels of the terminal neutrophil differentiation marker gelatinase $(27,28)$ and the neutrophil maturation marker Gr-1 $(29,30)$. In fact, G6pc3-/bone marrow aspirates contained a slightly higher proportion of colony-forming progenitor cells than did wild-type aspirates. When myeloid progenitor cells from 3 -week-old mice were allowed to differentiate in vitro in the presence of G-CSF, the granulocyte CFUs from both $\mathrm{G}_{6 p c 3^{-/}}$and wild-type littermates contained similar percentages of morphologically mature neutrophils, demonstrating that $G 6 p c 3^{-/-}$mice were not impaired in hematopoiesis or neutrophil maturation.

Another mechanism is ER stress. When cells experience conditions that alter the ER environment, such as glucose deprivation, protein folding can be dramatically affected, leading to the accumulation of unfolded proteins in the ER. Such alterations in ER homeostasis activate a series of signal transduction cascades collectively termed the UPR and characterized by the induction of expression of ER chaperones (20-23, 32, 33). A major function of the ER chaperones is to promote protein folding by preventing misfolding or aggregation $(20,21)$. However, prolonged activation of the UPR is proapoptotic $(32,33)$ and contributes to the pathogenesis of many diseases, including the neutrophil dysfunction that characterizes GSD-Ib (44). Neutrophils are a first line of defense against bacterial or fungal infection and originate in the bone marrow, which is the primary site of maturation and development of hematopoietic cells (25). We showed that during thioglycollate-induced peritonitis in $66 p c 3^{-/-}$mice, the expression of the ER chaperones, known as GRPs, was significantly increased in both neutrophils and bone marrow (Figure 7). Moreover, the G6pc3 $3^{-/-}$neutrophils exhibited a marked increase in apoptotic cell numbers evidenced by an increase
(17). Therefore, knocking out G6Pase- $\beta$ also significantly reduces the efficiency of G6P transport into the ER. The resulting low G6P concentration in the ER lumen not only deprives the ER of G6P hydrolase activity, but also affects another major metabolic pathway in neutrophils: the endoluminal pentose phosphate pathway, which generates NADPH $(45,46)$. The NADPH within the lumen of the ER is important for in vivo function of $11 \beta$-hydroxysteroid dehydrogenase-1 (47), an ER-associated oxoreductase that generates cortisol and/or corticosterone locally to modulate immune responses. The first 2 steps of this pathway are catalyzed by hexose-6-phosphate dehydrogenase (H6PDH; ref. 45). If this pathway is critical to neutrophil function, impairing $\mathrm{H} 6 \mathrm{PDH}$ function should yield a similar phenotype to the G6pc3 $3^{-1-}$ mouse strain. An H6PDH-deficient mouse line was reported recently (46), but neutrophil function was not addressed. It would be interesting to see if this pathway is also affected.

In conclusion, we have shown that G6Pase- $\beta$ inactivation led to neutrophil dysfunction in mice, which was most visible when the animals were subjected to immune stress. The mutation mimicked the myeloid dysfunction of G6PT-deficient GSD-Ib mice, tying the underlying mechanism to G6P transport to and metabolism within the ER lumen. Our findings highlight the critical role G6P plays in the ER of neutrophils during an immune response as well as its relationship to ER stress and neutrophil apoptosis in G6Pase- $\beta$ deficiency. It will be of interest to see whether G6Pase- $\beta$ mutations are associated with diseases in which intermittent neutropenia or neutrophil dysfunction emerges in the presence of stress or immune challenge.

\section{Methods} terial artificial chromosome (BAC) library (Invitrogen) from the $129 / \mathrm{SvJ}$
Gene targeting. The murine G6Pase- $\beta$ cDNA probe was used to screen a bac- 
mouse strain. The G6pc3 gene is a single-copy gene consisting of 6 exons. The targeting vector (Figure 1A) was designed to disrupt exons III, IV, and $\mathrm{V}$ using a neo- and HSV-tk-containing plasmid, pPNT/DL, obtained from P.L. Schwartzberg (National Human Genome Research Institute, Bethesda, Maryland, USA). The genes encoding for neo and HSV-tk are under the control of the phosphoglycerate kinase 1 promoter, and the neo gene is flanked by loxP sites. The vector was constructed in 2 steps. First, a $4-\mathrm{kb}$ G6Pase- $\beta$ BamHI-BamHI fragment containing exon VI and its 3' flanking region was isolated by PCR from the BAC clone and ligated into the BamHI linearized $\mathrm{pPNT} / \mathrm{DL}$ plasmid. The appropriate orientation of the resulting pPNT/DL-G6Pase- $\beta-B B$ construct was verified by restriction endonuclease mapping and DNA sequencing. Second, in parallel, a 4.9-kb BamHI-SanDI fragment of the G6pc3 gene containing exons I and II and approximately $2.6 \mathrm{~kb}$ of the $5^{\prime}$ flanking region of the G6pc3 gene was isolated by PCR using primers that had an additional NotI site at the $5^{\prime}$ end and a SalI site at the $3^{\prime}$ end. This fragment was then ligated into the NotI-Sall linearized plasmid $\mathrm{pPNT} / \mathrm{DL}-\mathrm{G} 6 \mathrm{Pase}-\beta-\mathrm{BB}$. The resulting targeting vector was verified by restriction endonuclease mapping and DNA sequencing.

The NotI linearized construct DNA was introduced into J1 ES cells by electroporation, the cells with the homologous recombination were selected with G418 and gancyclovir (24), and the disrupted G6pc3 gene was confirmed in these clones by Southern blotting of EcoRV-digested genomic DNA. Two probes, one corresponding to a G6pc3-specific 5' region (500 bp) lying $1 \mathrm{~kb}$ upstream of the $5^{\prime}$ BamHI site and the other to a G6pc3-specific 3' region (500 bp) lying $1.5 \mathrm{~kb}$ downstream of the 3' BamHI site, were used to confirm the mutation. In order to create the knockout mouse strains, 2 independently G6pc3-targeted ES cell clones, A184 and A206, were injected into C57BL/6 blastocysts and implanted into the uterine horns of pseudopregnant $\mathrm{FVB} / \mathrm{N}$ foster mice. Two lines of mice carrying the G6pc3-null mutation were selected. Chimeric mice were mated with C57BL/6 mice, and germline transmission of the G6pc3null allele in F1 heterozygous mice was confirmed by PCR analysis of tail DNA. Heterozygous animals were interbred to generate homozygous F2 (G6pc3-1-) mice, which were genotyped by PCR analysis on genomic tail DNA using the wild-type specific primer EIVs/EVas (5'-GCCTGGCTTATTGTACCTTC-3' and 5'-CCCAGTGTAAAGAGAGTCCA-3') and targeted locus-specific primers Neo1s/Neo1as (5'-TGGGATCGGCCATTGAACAA- $3^{\prime}$ and $5^{\prime}$-AAGCGGCCATTTTCCACCAT- ${ }^{\prime}$ ') and Neo2s/ EVIas (5' -GCCTTCTTGACGAGTTCTTC- 3 ' and $5^{\prime}$-TTCACACCACTTGGAAGCCA-3'). All animal studies were conducted under an animal protocol approved by the NICHD Animal Care and Use Committee.

Phosphobydrolase, Northern blot, real-time PCR, Western blot, hematological, and serum analyses. Microsome isolation and phosphohydrolase assays were determined essentially as described previously $(11,17)$. Reaction mixtures $(100 \mu \mathrm{l})$ contained $50 \mathrm{mM}$ cacodylate buffer, $\mathrm{pH} 6.5$, and $10 \mathrm{mM} \mathrm{G6P}$, and appropriate amounts of microsomal preparations were incubated at $37^{\circ} \mathrm{C}$ for 10 minutes. Nonspecific phosphatase activity was estimated by preincubating disrupted microsomal preparations at $\mathrm{pH} 5$ for 10 minutes at $37^{\circ} \mathrm{C}$ in order to inactivate the acid labile G6Pase- $\beta$. For immunodetection of the G6Pase $\beta$ protein, a rabbit antibody raised against a human G6Pase- $\beta$ peptide (48) that recognizes murine G6Pase- $\beta$ efficiently was used.

Total RNAs were isolated using TRIzol Reagent (Invitrogen) and poly $(\mathrm{A})^{+}$RNA was obtained by oligo(dT)-cellulose chromatography. RNA was fractionated by electrophoresis through $1.2 \%$ agarose gels containing $2.2 \mathrm{M}$ formaldehyde, and the filters were hybridized to a uniformly labeled G6Pase- $\beta$, G6PT, or $\beta$-actin riboprobe.

mRNA expression was quantified by real-time RT-PCR, in triplicate, in an Applied Biosystems 7300 Real-Time PCR System using gene-specific TaqMan Gene Expression Assays (Applied Biosystems) and then normalized to $\beta$-actin RNA. The following probes were used, all purchased from Applied
Biosystems: G6Pase- $\beta$, Mm00616234_m1; G6PT, Mm00484574_m1; GRP78, Mm00517691_m1; GRP170, Mm00491278_g1, caspase-3, Mm00438045_m1, and $\beta$-actin, Mm00607939_s1.

For Western blot analysis, peritoneal neutrophils and bone marrow aspirate lysates or microsomal preparations of brain, muscle, and testis were resolved by electrophoresis through a $10 \%$ or $16 \%$ polyacrylamideSDS gel and trans-blotted onto polyvinylidene fluoride membranes (Millipore). The membranes were incubated with a rabbit polyclonal antibody against G6Pase- $\beta$ (48), GRP170 (49), or caspase-3 (Cell Signaling Technology); a goat polyclonal antibody against a gelatinase peptide (Santa Cruz Biotechnology Inc.); a mouse monoclonal antibody against KDEL (Stressgen Biotechnologies) or $\beta$-actin (Santa Cruz Biotechnology Inc.); or a rat monoclonal antibody against Ly-6G/Gr-1 (BD Biosciences - Pharmingen). The membranes were then incubated with the respective horseradish peroxidase-conjugated secondary antibody obtained from Pierce Biotechnology. The immunocomplex was visualized using the SuperSignal West Pico Chemiluminescent substrate from Pierce Biotechnology.

Blood samples were collected from the tail vein using EDTA-containing CAPIJECT tubes (Terumo Medical) for the differential leukocyte counts. Manual 200-cell leukocyte differential counts of peripheral blood cells were performed on Hema 3-stained (Fischer Scientific) smears (26). Serum glucose, total cholesterol, and uric acid were analyzed using kits obtained from Thermo Electron, triglycerides were analyzed using a kit from SigmaAldrich, and lactic acid was analyzed using a kit from Trinity Biotech.

Respiratory burst, chemotaxis, and intracellular $\mathrm{Ca}^{2+}$ measurements. Neutrophils were isolated from thioglycollate-stimulated peritonea of 6- to 7 -week-old mice as described previously (26). The respiratory burst of neutrophils was monitored by luminally amplified chemiluminescence using the LumiMax Superoxide Anion Detection kit (Stratagene) and Victor Light 1420 Luminescence counter (PerkinElmer). The assay mixture $(0.2 \mathrm{ml})$ contained $200 \mu \mathrm{M}$ luminol, $250 \mu \mathrm{M}$ enhancer, and $2 \times 10^{5}$ neutrophils in HBSS. Neutrophils were activated with $200 \mathrm{ng} / \mathrm{ml}$ PMA (SigmaAldrich) in HBSS or with HBSS alone as a control.

Neutrophil chemotaxis in response to fMLP (Sigma-Aldrich), MIP-2 (PeproTech Inc.), or KC (PeproTech Inc.) was performed in 48-well chambers (NeuroProbe) using polyvinylpyrrolidone-free polycarbonate membranes with $3-\mu \mathrm{m}$ pores as described previously $(26,50)$. Lower chambers contained chemoattractant dissolved in HEPES-buffered RPMI medium containing $1 \%$ BSA (RPMI-BSA). Upper chambers contained $1 \times 10^{5}$ neutrophils in $50 \mu \mathrm{l}$ RPMI-BSA. After incubation at $37^{\circ} \mathrm{C}$ for 60 minutes, the membrane was removed, rinsed with PBS, fixed, and stained with Hema 3. Cells were counted in 6 randomly selected fields (original magnification, $\times 400$ ), and mean values were reported.

For intracellular $\mathrm{Ca}^{2+}$ concentration measurements, neutrophils $\left(10^{6}\right.$ cells) in $100 \mu \mathrm{l}$ of $1 \times$ HBSS containing $10 \mathrm{mM}$ HEPES were loaded with FLIPER calcium 3 assay kit component A (Molecular Devices) in a poly-L-lysine-coated 96-well plate (Greiner Bio-One). The plate was loaded into the reading chamber of a Flexstation Fluorimeter (Molecular Devices) set at $37^{\circ} \mathrm{C}$. The appropriate ligands were added to the plate robotically, and the fluorescence intensities were recorded every 0.5 seconds as the relative ratio of fluorescence emitted at $515 \mathrm{~nm}$ following excitation at $485 \mathrm{~nm}$.

Hematopoietic progenitor cell assays. Bone marrow cells from femoral and tibia bones were harvested by flushing with $3 \mathrm{ml}$ Iscove's modified Dulbecco's medium containing $2 \%$ fetal bovine serum. Progenitor cells were assayed in semisolid agar cultures by plating $2 \times 10^{4}$ bone marrow cells in $1 \mathrm{ml}$ of methylcellulose media (MethoCult M3231; StemCell Technologies Inc.) supplemented with recombinant murine cytokines G-CSF $(10 \mathrm{ng} / \mathrm{ml})$, GM-CSF (10 ng/ml), and M-CSF (2.5 ng/ml; all from R\&D Systems). The number of colonies larger than 50 cells was counted on days 7-9. 
Bacterial challenge. Log-phase E. coli (ATCC 25922) resuspended in PBS were injected into the peritonea of wild-type or $\mathrm{G} 6 \mathrm{pc} 3^{-/-}$mice at $4 \times 10^{7} \mathrm{CFU} / 30$ $\mathrm{g}$ body weight. The survival of the infected mice was monitored constantly throughout the experiments. At 5 hours after infection, blood samples were collected, and viable bacterial counts were determined by plating serial dilutions of samples on Luria-Bertani plates for culture and counting.

Apoptosis and caspase-3 activity assays. Apoptotic neutrophils were scored by annexin $\mathrm{V}$ surface staining. Briefly, thioglycollate-elicited peritoneal neutrophils $\left(1 \times 10^{6} \mathrm{cells} / \mathrm{ml}\right)$ were stained with FITC-conjugated annexin V using the annexin V-FITC apoptosis detection kit (BioVision) according to the manufacturer's instructions. After incubation for 10 minutes in the dark, cells were plated onto the glass slides by cytospin and then fixed with $2 \%(\mathrm{v} / \mathrm{v})$ formaldehyde solution for 15 minutes. Nuclei were stained with DAPI (Sigma-Aldrich). Fluorescence was visualized using the Axioskop2 plus fluorescence microscope (Zeiss). The images were digitalized with AxioVision software (version 4.3; Zeiss).

The DEVD-cleaving activity of active caspase- 3 was measured using the labeled Asp-Glu-Val-Asp-p-nitroanilide (DEVD-pNA) as substrate and the caspase- 3 colorimetric assay kit from BioVision according to the manufac- turer's protocol. The pNA light emission was quantified using a microtiter plate reader at a wavelength of $405 \mathrm{~nm}$.

Statistics. Statistical analysis using unpaired Student's $t$ test was performed with GraphPad Prism 4.0 (GraphPad Software). The log-rank test, used to analyze survival data, was performed with SPSS 14.0 for Windows (SPSS Inc.). Values were considered statistically significant at $P<0.05$.

\section{Acknowledgments}

We thank Anil B. Mukherjee for critical reading of the manuscript and John R. Subjeck for his gift of antiserum against GRP170. This research was supported in part by the Intramural Research Program of the NICHD, NIH.

Received for publication September 22, 2006, and accepted in revised form December 26, 2006.

Address correspondence to: Janice Y. Chou, Building 10, Room 9D42, NIH, Bethesda, Maryland 20892, USA. Phone: (301) 4961094; Fax: (301) 402-6035; E-mail: chouja@mail.nih.gov.
1. Chou, J.Y., Matern, D., Mansfield, B.C., and Chen, Y.T. 2002. Type I glycogen storage diseases: disorders of the glucose-6-phosphatase complex. Curr. Mol. Med. 2:121-143

2. Chou, J.Y., and Mansfield, B.C. 2003. Glucose-6phosphate transporter: the key to glycogen storage disease type $\mathrm{Ib}$. In Membrane transporter diseases. S. Broer and C.A. Wagner, editors. Springer. New York, New York, USA. 191-205.

3. Beaudet, A.L., Anderson, D.C., Michels, V.V., Arion, W.J., and Lange, A.J. 1980. Neutropenia and impaired neutrophil migration in type $1 \mathrm{~B}$ glycogen storage disease. J. Pediatr. 97:906-910.

4. Gitzelmann, R., and Bosshard, N.U. 1993. Defective neutrophil and monocyte functions in glycogen storage disease type 1b: a literature review. Eur. J. Pediatr. 152(Suppl.):S33-S38

5. Rothbaum, R., et al. 2002. Shwachman-Diamond syndrome: report from an international conference. J. Pediatr. 141:266-270.

6. Badolato, R., Fontana, S., Notarangelo, L.D., and Savoldi, G. 2004. Congenital neutropenia: advances in diagnosis and treatment. Curr. Opin. Allergy Clin. Immunol. 4:513-521.

7. Berliner, N., Horwitz, M., and Loughran, T.P., Jr. 2004. Congenital and acquired neutropenia. Hematology Am. Soc. Hematol. Educ. Program. 63-79.

8. Zeidler, C. 2005. Congenital neutropenias. Hematology. 10 (Suppl. 1):306-311.

9. Pan, C.J., Lei, K.J., Annabi, B., Hemrika, W., and Chou, J.Y. 1998. Transmembrane topology of glucose-6-phosphatase. J. Biol. Chem. 273:6144-6148.

10. Pan, C.J., Lin, B., and Chou, J.Y. 1999. Transmembrane topology of human glucose-6-phosphate transporter. J. Biol. Chem. 274:13865-13869.

11. Lei, K.J., et al. 1996. Glucose-6-phosphatase dependent substrate transport in the glycogen storage disease type 1a mouse. Nat. Genet. 13:203-209.

12. Hiraiwa, H., Pan, C.-J., Lin, B., Moses, S.W., and Chou, J.Y. 1999. Inactivation of the glucose-6-phosphate transporter causes glycogen storage disease type 1b. J. Biol. Chem. 274:5532-5536.

13. Nordlie, R.C., and Sukalski, K.A. 1985. Multifunctional glucose-6-phosphatase: a critical review. In The enzymes of biological membranes. 2nd Edition. A.N. Martonosi, editor. Springer. New York, New York, USA. 349-398.

14. Pan, C.J., Kei, K.J., Chen, H., Ward, J.M., and Chou, J.Y. 1998. Ontogeny of the murine glucose-6-phosphatase system. Arch. Biochem. Biophys. 358:17-24.

15. Lin, B., Annabi, B., Hiraiwa, H., Pan, C.J., and
Chou, J.Y. 1998. Cloning and characterization of cDNAs encoding a candidate glycogen storage disease type $1 \mathrm{~b}$ protein in rodents. J. Biol. Chem. 273:31656-31670

16. Guionie, O., Clottes, E., Stafford, K., and Burchell, A. 2003. Identification and characterization of a new human glucose-6-phosphatase isoform. FEBS Lett. 551:159-164.

17. Shieh, J.J., Pan, C.J., Mansfield, B.C., and Chou, J.Y. 2003. Glucose-6-phosphate hydrolase, widely expressed outside the liver, can explain age-dependent resolution of hypoglycemia in glycogen storage disease type Ia. J. Biol. Chem. 278:47098-47103.

18. Ghosh, A., Shieh, J.J., Pan, C.J., and Chou, J.Y. 2004. Histidine-167 is the phosphate acceptor in glucose- 6 -phosphatase- $\beta$ forming a phosphohistidine-enzyme intermediate during catalysis. J. Biol. Chem. 279:12479-12483.

19. Ghosh, A., et al. 2002. The catalytic center of glucose-6-phosphatase: His ${ }^{176}$ is the nucleophile forming the phosphohistidine-enzyme intermediate during catalysis. J. Biol. Chem. 277:32837-32842.

20. Kuznetsov, G., and Nigam, S.K. 1998. Folding of secretory and membrane proteins. N. Engl. J. Med. 339:1688-1695.

21. Lee, A.S. 2001. The glucose-regulated proteins: stress induction and clinical applications. Trends Biochem. Sci. 26:504-510.

22. Kleizen, B., and Braakman, I. 2004. Protein folding and quality control in the endoplasmic reticulum. Curr. Opin. Cell Biol. 16:343-349.

23. Zhang, K., and Kaufman, R.J. 2006. The unfolded protein response: a stress signaling pathway critical for health and disease. Neurology. 66(Suppl. 1):S102-S109.

24. Tybulewicz, V.L.J., Crawford, C.E., Jackson, P.K., Bronson, R.T., and Mulligan, R.C. 1991. Neonatal lethality and lymphopenia in mice with a homozygous disruption of the c-abl proto-oncogene. Cell. 65:1153-1163.

25. Sieff, C.A., Nathan, D.G., and Clark, S.C. 1998. The anatomy and physiology of hematopoiesis. In Hematology of infancy and childhood. Volume 1. 5th edition. D.G. Nathon and S.H. Orkin, editors. W.B. Saunders. Philadelphia, Pennsylvania, USA. 161-236.

26. Chen, L.Y., et al. 2003. Impaired glucose homeostasis, neutrophil trafficking and function in mice lacking the glucose-6-phosphate transporter. Hum. Mol. Genet. 12:2547-2558.

27. Borregaard, N., Sehested, M., Nielsen, B.S., Sengelov, H., and Kjeldsen, L. 1995. Biosynthesis of granule proteins in normal human bone marrow cells. Gelatinase is a marker of terminal neutrophil differentiation. Blood. 85:812-817.

28. Faurschou, M., and Borregaard, N. 2003. Neutrophil granules and secretary vesicles in inflammation. Microbes Infect. 5:1317-1327.

29. Hestdal, K.K., et al. 1991. Characterization and regulation of RB6-8C5 antigen expression on murine bone marrow cells. J. Immunol. 147:22-28.

30. Fleming, T.J., Fleming, M.L., and Malek, T.R. 1993. Selective expression of Ly-6G on myeloid lineage cells in mouse bone marrow. RB6-8C5 mAb to granulocyte-differentiation antigen (Gr-1) detects members of the Ly- 6 family. J. Immunol. 151:2399-2408.

31. Stevens, F.J., and Argon, Y. 1999. Protein folding in the ER. Semin. Cell Dev. Biol. 10:443-454.

32. Xu, C., Bailly-Maitre, B., and Reed, J.C. 2005. Endoplasmic reticulum stress: cell life and death decisions. J. Clin. Invest. 115:2656-2664. doi:10.1172/ JCI26373.

33. Schroder, M., and Kaufman, R.J. 2005. The mammalian unfolded protein response. Annu. Rev. Biochem. 74:739-789.

34. Vance, J.E., and Steenbergen, R. 2005. Metabolism and functions of phosphatidylserine. Prog. Lipid Res. 44:207-234.

35. Lavrik, I.N., Golks, A., and Krammer, P.H. 2005. Caspases: pharmacological manipulation of cell death. J. Clin. Invest. 115:2665-2672. doi:10.1172/ JCI26252.

36. Siegel, R.M. 2006. Caspases at the crossroads of immune-cell life and death. Nat. Rev. Immunol. 6:308-317.

37. Daleke, D.L. 2003. Regulation of transbilayer plasma membrane phospholipid asymmetry. J. Lipid Res. 44:233-242.

38. Blankenberg, F.G. 2004. Recent advances in the imaging of programmed cell death. Curr. Pharm. Des. 10:1457-1467.

39. Kim, S.Y., et al. 2006. Bone-marrow derived cells require a functional glucose-6-phosphate transporter for normal myeloid functions. J. Biol. Chem. 281:28794-28801.

40. Silva, A.J., et al. 1997. Mutant mice and neuroscience: recommendations concerning genetic background. Banbury Conference on Genetic Background in Mice. Neuron. 19:755-759.

41. Wolfer, D.P., Crusio, W.E., and Lipp, H.P. 2002. Knockout mice: simple solutions to the problems of genetic background and flanking genes. Trends Neurosci. 25:336-340.

42. Liu, B., Koo, G.C., Yap, E.H., Chua, K.L., and Gan, 
Y.H. 2001. Model of differential susceptibility to mucosal Burkholderia pseudomallei infection. Infect. Immun. 70:504-511.

43. Matsutani, T., Anantha-Samy, T.S., Kang, S.C., Bland, K.I., and Chaudry, I.H. 2005. Mouse genetic background influences severity of immune responses following trauma-hemorrhage. Cytokine. 30:168-176.

44. Kuijpers, T.W., et al. 2003. Apoptotic neutrophils in the circulation of patients with glycogen storage disease type 1b (GSD1b). Blood. 101:5021-5024.
45. Hewitt, K.N., Walker, E.A., and Stewart, P.M. 2005. Minireview: hexose-6-phosphate dehydrogenase and redox control of 11beta-hydroxysteroid dehydrogenase type 1 activity. Endocrinology. 146:2539-2543.

46. Lavery, G.G., et al. 2006. Hexose-6-phosphate dehydrogenase knock-out mice lack 11 beta-hydroxysteroid dehydrogenase type 1-mediated glucocorticoid generation. J. Biol. Chem. 281:6546-6551.

47. Tomlinson, J.W., et al. 2004. 11beta-hydroxysteroid dehydrogenase type 1: a tissue-specific regulator of glucocorticoid response. Endocr. Rev. 25:831-866. 48. Shieh, J.J., Pan, C.J., Mansfield, B.C., and Chou, J.Y. 2004. A potential new role for muscle in blood glucose homeostasis. J. Biol. Chem. 279:26215-26219.

49. Lin, H.Y., et al. 1993. The $170-\mathrm{kDa}$ glucose-regulated stress protein is an endoplasmic reticulum protein that binds immunoglobulin. Mol. Biol. Cell. 4:1109-1119.

50. Gao, J.L., Lee, E.J., and Murphy, P.M. 1999. Impaired antibacterial host defense in mice lacking the $\mathrm{N}$-formylpeptide receptor. J. Exp. Med. 189:657-662. 